\title{
A CLASS OF ARITHMETICAL FUNCTIONS IN SEVERAL VARIABLES WITH APPLICATIONS TO CONGRUENCES
}

\author{
BY \\ ECKFORD COHEN
}

1. Introduction. Let $r$ represent a positive integer. In this paper we generalize the concepts of even and primitive function $(\bmod r)$ to functions of several variables. It is recalled that a (complex-valued) function $f_{r}(n)$ is even $(\bmod r)$ if $f_{r}(n)=f_{r}((n, r))$ for all integral $n$, and is primitive $(\bmod r)$ if $f_{r}(n)=f_{r}(\gamma(n, r))$ for all $n$, where $\gamma(r)$ denotes the core of $r$ and $\gamma(n, r)=\gamma((n, r))$. (The core $\gamma(r)$ is defined to be the product of the distinct prime divisors of $r, \gamma(1)=1$.)

Let $n_{1}, \cdots, n_{k}$ denote $k$ integral variables. Then a complex-valued function $f_{r}\left(n_{1}, \cdots, n_{k}\right)$ will be defined to be a (relatively) even function of $n_{1}, \cdots, n_{k}(\bmod r)$ provided $f_{r}\left(n_{1}, \cdots, n_{k}\right)=f_{r}\left(\left(n_{1}, r\right), \cdots,\left(n_{k}, r\right)\right)$ for all $n_{i}(i=1, \cdots, k)$; if $f_{r}\left(n_{1}, \cdots, n_{k}\right)=f_{r}\left(\gamma\left(n_{1}, r\right), \cdots, \gamma\left(n_{k}, r\right)\right)$ for all $n_{i}$, then $f_{r}\left(n_{1}, \cdots, n_{k}\right)$ will be called a primitive function of $n_{1}, \cdots, n_{k}(\bmod r)$. As alternative definitions, one may say that $f_{r}\left(n_{1}, \cdots, n_{k}\right)$ is even (primitive) as a function of $n_{1}, \cdots, n_{k}(\bmod r)$ provided it is even (primitive) as a function of each $n_{i}(\bmod r)$. Clearly the class of even functions $(\bmod r)$ contains the class of primitive functions $(\bmod r)$ as a subclass.

The above definitions will be specialized in the following manner. If there exists an even function $F_{r}(n)$ of $n(\bmod r)$ such that $f_{r}\left(n_{1}, \cdots, n_{k}\right)$ $=F_{r}\left(\left(n_{1}, \cdots, n_{k}\right)\right)$ for all $n_{i}(i=1, \cdots, k)$, then $f_{r}\left(n_{1}, \cdots, n_{k}\right)$ will be called a totally even function of $n_{1}, \cdots, n_{k}(\bmod r)$; if $f_{r}\left(n_{1}, \cdots, n_{k}\right)$ $=F_{r}\left(\gamma\left(n_{1}, \cdots, n_{k}\right)\right)$ for all $n_{i}$, then $f_{r}\left(n_{1}, \cdots, n_{k}\right)$ will be termed a totally primitive function $(\bmod r)$. Alternatively, $f_{r}\left(n_{1}, \cdots, n_{k}\right)$ may be defined to be totally even (totally primitive) in the variables $n_{1}, \cdots, n_{k}$, provided it is an even (primitive) function $(\bmod r)$ in the single variable $\left(n_{1}, \cdots, n_{k}\right)$.

For simplicity the discussion in this paper will be largely confined to the case $k=2$, that is, to the case of functions $f_{r}(m, n)$ of two integral variables $m, n(\bmod r)$. The remainder of this section is devoted to a brief sketch of the content of the paper.

In $\$ 2$ we collect for later reference a number of results most of which are known. In $\S \S 3$ and 4 we obtain trigonometric characterizations of the even and totally even functions $(\bmod r)$, (Theorems 1 and 2 , respectively). More precisely, Fourier expansions in terms of functions involving Ramanujan's sum $c_{r}(n)$ are deduced for even functions $f_{r}(m, n)$. These expansions are applied in $\$ 5$ to obtain formulas for the number of solutions of pairs of congruences $(\bmod r)$ of which at least one is bilinear. Similar applications to

Presented to the Society, November 29, 1958; received by the editors July 11, 1959. 
pairs of linear congruences are developed in $\S 6$. The notion of Cauchy product $(\bmod r)$ is strongly emphasized in this section (cf. [4]). Specifically, the Cauchy product $(\bmod r)$ of two functions $f_{r}(m, n), g_{r}(m, n)$ is defined by

$$
h_{r}(m, n)=\sum_{m \equiv a+b(\bmod r) ; n \equiv a^{\prime}+b^{\prime}(\bmod r)} f_{r}\left(a, a^{\prime}\right) g_{r}\left(b, b^{\prime}\right),
$$

where $a, b, a^{\prime}, b^{\prime}$ range over integers $(\bmod r)$ such that $m \equiv a+b(\bmod r)$, $n \equiv a^{\prime}+b^{\prime}(\bmod r)$.

In $\$ \S 7$ and 8 we obtain trigonometric characterizations of the primitive and totally primitive functions $(\bmod r)$, respectively. As an application, in Theorem $14(\S 7)$ a simple arithmetical formula is obtained for the number of solutions $\omega_{r}(m, n)$ of the congruences,

$$
\begin{aligned}
m & \equiv u+x(\bmod r), \\
n & \equiv v+y(\bmod r),
\end{aligned}
$$

such that $(u, r)=(v, r)=(x, y, r)=1$. An analogous result is obtained in $\S 8$ (Corollary 18.1) for the number of solutions $\theta_{r}(m, n)$ of (1.1) such that $(u, v, r)=(x, y, r)=1$. As a corollary it follows that $\theta_{r}(m, n)>0$ for all values of $m, n, r$ (Corollary 18.2). Precise criteria for the vanishing of $\omega_{r}(m, n)$ are deduced in Theorem $15(\$ 7)$.

In $\S 9$ we obtain analogues in two variables of some of Ramanujan's expansions of arithmetical functions in infinite series. The proofs are based on the finite Fourier expansions of certain even functions $(\bmod r)$. In the final section $(\$ 10)$ we generalize to $k$ variables several of the representations theorems proved earlier in the paper in the case $k=2$. The proofs are omitted.

2. Preliminaries. The Möbius and Euler functions will be denoted by $\mu(r)$ and $\phi(r)$, respectively. We use $J_{k}(r)$ to denote the Jordan function, defined to be the number of $k$-dimensional, integral vectors $X=\left\{x_{1}, \cdots, x_{k}\right\}$ in a reduced residue system $(\bmod k, r)$, that is, the number of $X, x_{i}(\bmod r)$, $i=1, \cdots, k$, such that $\left(\left(x_{i}\right), r\right) \equiv\left(\left(x_{1}, \cdots, x_{k}\right), r\right)=1[2, \S 2]$. We recall that

$$
J_{k}(r)=\sum_{d \mid r} d^{k} \mu\left(\frac{r}{d}\right)
$$

$$
\phi(r)=J_{1}(r)
$$

REMARK 1 . If $d \mid r$, then a reduced residue system $(\bmod k, r)$ can be decomposed into $J_{k}(r) / J_{k}(d)$ such systems $(\bmod k, d)$, [2, Lemma 7]. Consequently, in selecting a reduced residue system $(\bmod k, d)$ it is permissible to restrict the choice of $X$ to elements satisfying $\left(\left(x_{i}\right), r\right)=1$.

REMARK 2. A complete residue system $(\bmod k, r)$ is generated by the set $(r / d) X$ where $d$ ranges over the divisors of $r$, and for each $d, X$ ranges over a reduced residue system $(\bmod k, d),[2$, Lemma 1$]$.

Note, in case $k=1$, that these remarks relate to ordinary residue systems $(\bmod r)$. 
The symbol $e_{r}(n)$ is defined by $e_{r}(n)=\exp (2 \pi i n / r)$. We note the familiar property,

$$
\eta_{r}(n) \equiv \sum_{a(\bmod r)} e_{r}(n a)= \begin{cases}r & (r \mid n), \\ 0 & (r \nmid n) .\end{cases}
$$

The trigonometric sum of Ramanujan will be denoted by $c_{r}(n)$ :

$$
c_{r}(n)=\sum_{(x, r)=1} e_{r}(n x)
$$

the summation being over a reduced residue system $(\bmod r)$. The Ramanujan and Dedekind-Hölder evaluations (cf. $[5 ; 6]$ ) of $c_{r}(n)$ are given, respectively, by

$$
\begin{aligned}
c_{r}(n) & =\sum_{d \backslash(n, r)} d \mu\left(\frac{r}{d}\right), & \\
c_{r}(n) & =\frac{\phi(r) \mu(b)}{\phi(b)}, & \left(b=\frac{r}{(n, r)}\right) .
\end{aligned}
$$

We also recall the following additional properties of $c_{r}(n)$. Let $d_{1}$ and $d_{2}$ be divisors of $r$; then [1]

$$
\begin{aligned}
c_{d_{2}}\left(\frac{r}{d_{1}}\right) & =\frac{\phi\left(d_{2}\right)}{\phi\left(d_{1}\right)} c_{d_{1}}\left(\frac{r}{d_{2}}\right), \\
\sum_{d \mid r} c\left(\frac{r}{d}, d_{1}\right) c\left(\frac{r}{d_{2}}, d\right) & = \begin{cases}r & \left(d_{1}=d_{2}\right), \\
0 & \left(d_{1} \neq d_{2}\right) .\end{cases}
\end{aligned}
$$

As a special case of (2.7) one obtains $\left(d_{1}=1\right)$,

$$
\sum_{d \mid r} c(n, d)= \begin{cases}r & (r \mid n), \\ 0 & (r \nmid n) .\end{cases}
$$

The case $n=1$ of (2.8) yields the characteristic property of $\mu(r)$. A useful reformulation of (2.7) is given by (6.5).

The function $c_{r}(n)$ can be generalized $[2, \S 3]$ by placing

$$
c_{r}^{(k)}(n)=\sum_{\left(\left(x_{i}\right), r\right)=1} e_{r}\left(n\left(x_{1}+\cdots+x_{k}\right)\right),
$$

where the summation is over a reduced residue system $(\bmod k, r)$. Evidently $c_{r}(n)=c_{r}^{(1)}(n)$. Analogous to (2.4) and (2.5) we have

$$
\begin{aligned}
c_{r}^{(k)}(n) & =\sum_{d \mid(n, r)} d^{k} \mu\left(\frac{r}{d}\right), & \\
c_{r}^{(k)}(n) & =\frac{J_{k}(r) \mu(b)}{J_{k}(b)}, & \left(b=\frac{r}{(n, r)}\right) .
\end{aligned}
$$


REMARK 3. It will be noted that $c_{r}^{(k)}(n)$ is an even function of $n(\bmod r)$. We next restate a result of [2] in a slightly more general form.

LEMma 1. If $s \geqq 0$ and $\left(m_{0}, \cdots, m_{s}, r\right)=1$, then the number of solutions $\phi_{s}^{*}(n, r)$ of the congruence, $n \equiv m_{0} x_{0}+\cdots+m_{s} x_{s}(\bmod r)$ in $x_{i}(\bmod r), i$ $=0, \cdots, s$, such that $\left(x_{0}, \cdots, x_{s}, r\right)=1$, is given by $\phi_{s}^{*}(n, r)$ $=(r /(n, r))^{8} J_{s}((n, r))$.

Proof. If $r=r_{1} r_{2},\left(r_{1}, r_{2}\right)=1$, it follows easily by the Chinese Remainder Theorem that $\phi_{s}^{*}(n, r)=\phi_{s}^{*}\left(n, r_{1}\right) \phi_{s}^{*}\left(n, r_{2}\right)$. It therefore suffices to prove the lemma in case $r=p^{t}, p$ prime, $t>0$. In this case, one may assume that $p \nmid m_{0}$, let us say; the argument of [2, Theorem 5$]$ can then be applied to complete the proof.

REMARK 4. The function $\phi_{s}^{*}(n, r)$ defined in Lemma 1 is independent of the $m_{i}$, under the assumption that $\left(m_{0}, \cdots, m_{s}, r\right)=1$.

The function $c_{r}^{(k)}(n)$ can be generalized by writing

$$
c_{r}\left(m_{1}, \cdots, m_{k}\right)=\sum_{\left(\left(x_{i}\right), r\right)=1} e_{r}\left(m_{1} x_{1}+\cdots+m_{k} x_{k}\right) .
$$

By Remarks 3 and 4, however, it follows that

LeMma 2. If $m_{1}, \cdots, m_{k}$ are arbitrary integers, then

$$
c_{r}\left(m_{1}, \cdots, m_{k}\right)=c_{r}^{(k)}((m, r))=c_{r}^{(k)}(m),
$$

where $m=\left(m_{1}, \cdots, m_{k}\right)$.

Finally, we note a simple property of the greatest common divisor and least common multiple. Let $d, \delta$ be divisors of $r$. Then

$$
(d, \delta)\left[\frac{r}{d}, \frac{r}{\delta}\right]=r ;
$$

in particular, if $r=d \delta,(2.14)$ yields the familiar relation,

$$
(d, \delta)[d, \delta]=d \delta \text {. }
$$

3. Trigonometric representations of even functions $(\bmod r)$. In view of Remark 1 ( $\$ 2)$ we are justified in adopting the following convention, to be referred to by the symbol $\left({ }^{*}\right)$, when specifically required.

*Convention. In all summations over a reduced residue system ( $\bmod k, d)$ where $d \mid r$, it will be assumed that the elements $X$ comprising the system are also elements of a reduced residue system $(\bmod k, r)$.

We now prove the basic result of this paper.

THEOREM 1. If $f_{r}(m, n)$ is an even function of $m, n(\bmod r)$, then $f_{r}(m, n)$ has a representation of the form 


$$
f_{r}(m, n)=\sum_{d|r ; \delta| r} \alpha_{r}(d, \delta) c_{d}(m) c_{\delta}(n),
$$

where the (Fourier) coefficients $\alpha_{r}(d, \delta)$ are uniquely determined by the formula,

$$
\alpha_{r}(d, \delta)=\frac{1}{r^{2}} \sum_{D|r ; E| r} f_{r}\left(\frac{r}{D}, \frac{r}{E}\right) c_{D}\left(\frac{r}{d}\right) c_{E}\left(\frac{r}{\delta}\right),
$$

or by the equivalent formula

$$
\alpha_{r}(d, \delta)=\frac{1}{r^{2} \phi(d) \phi(\delta)} \sum_{a, b(\bmod r)} f_{r}(a, b) c_{d}(a) c_{\delta}(b) .
$$

Conversely every function of the form $(3.1)$ is even $(\bmod r)$.

Proof. As a periodic function $(\bmod r), f_{r}(m, n)$ has the Fourier expansion [7]

$$
f_{r}(m, n)=\sum_{x, y(\bmod r)} \beta_{r}(x, y) e_{r}(m x) e_{r}(n y),
$$

where the $\beta_{r}(x, y)$ are uniquely determined by

$$
\beta_{r}(x, y)=\frac{1}{r^{2}} \sum_{u, v(\bmod r)} f_{r}(u, v) e_{r}(-x u) e_{r}(-y v) .
$$

By Remark $2(\S 2)$ we may replace $x$ and $y$ in (3.4) and (3.5) by $x=(r / d) X$, $y=(r / \delta) Y,(X, d)=(Y, \delta)=1$, to get

$$
\begin{aligned}
f_{r}(m, n) & =\sum_{d|r ; \delta| r} \sum_{(X, d)=1 ;(Y, \delta)=1} \beta_{r}\left(\frac{r X}{d}, \frac{r Y}{\delta}\right) e_{d}(m X) e_{\delta}(n Y), \\
\beta_{r}\left(\frac{r X}{d}, \frac{r Y}{\delta}\right) & =\frac{1}{r^{2}} \sum_{w, v(\bmod r)} f_{r}(u, v) e_{r}\left(\frac{-r X u}{d}\right) e_{r}\left(\frac{-r Y v}{\delta}\right) .
\end{aligned}
$$

With $u=(r / D) U, v=(r / E) V,(U, D)=(V, E)=1$, (3.7) becomes $\beta_{r}\left(\frac{r X}{d}, \frac{r Y}{\delta}\right)$

$$
=\frac{1}{r^{2}} \sum_{D|r ; E| r} \sum_{(U, D)=1 ;(V, E)=1} f_{r}\left(\frac{r U}{D}, \frac{r V}{E}\right) e_{D}\left(\frac{-r X U}{d}\right) e_{E}\left(\frac{-r Y V}{\delta}\right) .
$$

However, since $f_{r}(m, n)$ and $c_{r}(n)$ are even $(\bmod r)$, it follows $\left(^{*}\right)$ that

$$
\beta_{r}(r X / d, r Y / \delta)
$$

$$
=\beta_{r}\left(\frac{r}{d}, \frac{r}{\delta}\right)=\frac{1}{r^{2}} \sum_{D|r ; E| r} f_{r}\left(\frac{r}{D}, \frac{r}{E}\right) c_{D}\left(\frac{r}{d}\right) c_{E}\left(\frac{r}{\delta}\right),
$$

and, by (3.6), that 


$$
f_{r}(m, n)=\sum_{d|r ; \delta| r} \beta_{r}\left(\frac{r}{d}, \frac{r}{\delta}\right) c_{d}(m) c_{\delta}(n) .
$$

Placing $\alpha_{r}(d, \delta)=\beta_{r}(r / d, r / \delta)$, (3.9) and (3.8) lead to (3.1) and (3.2).

To show that (3.3) and (3.2) are equivalent, denote the right member of (3.3) by $\alpha_{r}^{\prime}(d, \delta)$. Placing $a=(r / D) x, b=(r / E) y,(x, D)=(y, E)=1$, we obtain (Remark 2)

$$
\alpha_{r}^{\prime}(d, \delta)=\frac{1}{r^{2} \phi(d) \phi(\delta)} \sum_{D|r ; E| r} \sum_{(x, D)=1 ;(y, E)=1} f_{r}\left(\frac{r x}{D}, \frac{r y}{E}\right) c_{d}\left(\frac{r x}{D}\right) c_{\delta}\left(\frac{r y}{E}\right),
$$

so that by the evenness $(\bmod r)$ of $c_{r}(n)$ and $f_{r}(m, n)$, it follows $\left(^{*}\right)$ that

$$
\alpha_{r}^{\prime}(d, \delta)=\frac{1}{r^{2} \phi(d) \phi(\delta)} \sum_{D|r ; E| r} \phi(D) \phi(E) f_{r}\left(\frac{r}{D}, \frac{r}{E}\right) c_{d}\left(\frac{r}{D}\right) c_{\delta}\left(\frac{r}{E}\right) .
$$

Application of (2.6) gives $\alpha_{r}^{\prime}(d, \delta)=\alpha_{r}(d, \delta)$, and (3.3) is proved.

The converse part of the theorem follows by noting that $c_{d}(n)$ is even $(\bmod r)$ for each divisor $d$ of $r$. Thus Theorem 1 is proved.

It will be observed that the relation between (3.1) and (3.2) is an inversion formula for the (even) functions $f_{r}(m, n)$ and $\alpha_{r}(m, n)$. In particular, if we place $\alpha_{r}(d, \delta)=g_{r}(d, \delta)$, we obtain, in case $d=r /(m, r), \delta=r /(n, r)$,

Corollary 1.1. If

$$
f_{r}(m, n)=\sum_{d|r ; \delta| r} g_{r}(d, \delta) c_{d}(m) c_{\delta}(n)
$$

then

$$
g_{r}\left(\frac{r}{(m, r)}, \frac{r}{(n, r)}\right)=\frac{1}{r^{2}} \sum_{d|r ; \delta| r} f_{r}\left(\frac{r}{d}, \frac{r}{\delta}\right) c_{d}(m) c_{\delta}(n) .
$$

4. Trigonometric representations of totally even functions $(\bmod r)$. We shall show in this section that if $f_{r}(m, n)$ is assumed totally even (mod $r$ ) then Theorem 1 can be stated in a much simpler form. For this purpose we introduce the following additional notation. Let $J(r)=J_{2}(r), C_{r}(n)=c_{r}^{(2)}(n)$, so that by Lemma $2, c_{r}(m, n)=C_{r}((m, n))$. In particular, $c_{r}(m, n)$ is totally even $(\bmod r)$; moreover, by $(2.10)$ and $(2.11)$ we have

$$
\begin{aligned}
& c_{r}(m, n)=\sum_{d \mid(m, n, r)} d^{2} \mu\left(\frac{r}{d}\right), \\
& c_{r}(m, n)=\frac{J(r) \mu(b)}{J(b)},
\end{aligned}
$$$$
\left(b=\frac{r}{(m, n, r)}\right)
$$

We also have the following analogue of (2.6). 
LEMma 3. If $d|r, \delta| r$, then

$$
C_{\delta}\left(\frac{r}{d}\right)=\frac{J(\delta)}{J(d)} C_{d}\left(\frac{r}{\delta}\right) .
$$

Proof. Place $t_{1}=\delta /(\delta, r / d), t_{2}=d /(d, r / \delta)$, so that $t_{1}=t_{2}$. Hence by (4.2),

$$
C_{\delta}\left(\frac{r}{d}\right)=\frac{J(\delta) \mu\left(t_{1}\right)}{J\left(t_{1}\right)}=\frac{J(\delta) \mu\left(t_{2}\right)}{J\left(t_{2}\right)}=\frac{J(\delta)}{J(d)} \cdot C_{d}\left(\frac{r}{\delta}\right) .
$$

The lemma is proved.

We next deduce a fundamental relation between $c_{r}(m, n)$ and the ordinary Ramanujan sums.

Lemma 4.

$$
c_{r}(m, n)=\sum_{d|r, \delta| r ;[d, \delta]=r} c_{d}(m) c_{\delta}(n) .
$$

Proof. By (2.12)

$$
c_{r}(m, n)=\sum_{(x, y, r)=1} e_{r}(m x+n y) .
$$

Placing $s=(r / d) X, y=(r / \delta) Y,(X, d)=(Y, \delta)=1$, we have $\left(^{*}\right)$ by Remark 2,

$$
\begin{aligned}
c_{r}(m, n) & =\sum_{d|r, \delta| r ;(r / d, r / \delta)=1} \sum_{(X, d)=1 ;(Y, \delta)=1} e_{r}\left(\frac{m r X}{d}\right) e_{r}\left(\frac{n r Y}{\delta}\right) \\
& =\sum_{d|r, \delta| r ;(r / d, r / \delta)=1} c_{d}(m) c_{\delta}(n) .
\end{aligned}
$$

The lemma follows by (2.14).

THEOREM 2. If $f_{r}(m, n)=F_{r}((m, n))$ is a totally even function of $m, n(\bmod r)$, then $f_{r}(m, n)$ has a representation of the form,

$$
f_{r}(m, n)=\sum_{d \mid r} \alpha_{r}(d) c_{d}(m, n),
$$

where $\alpha_{r}(d)$ is uniquely determined by the formula,

$$
\alpha_{r}(d)=\frac{1}{r^{2}} \sum_{\delta \mid r} F_{r}\left(\frac{r}{\delta}\right) C_{\delta}\left(\frac{r}{d}\right),
$$

or by the equivalent formula,

$$
\alpha_{r}(d)=\frac{1}{r^{2} J(d)} \sum_{a, b(\bmod r)} f_{r}(a, b) c_{d}(a, b) .
$$

Conversely, every function (4.5) is totally even $(\bmod r)$.

Proof. We deduce Theorem 2 from Theorem 1 under the assumption that 
$f_{r}(m, n)$ is totally even $(\bmod r)$. Let $\alpha_{r}(d)$ be a function defined by (4.6) for all divisors $d$ of $r$, and place $\beta_{r}(d)=\alpha_{r}(r / d)$. By Theorem $1, f_{r}(m, n) \equiv F_{r}((m, n))$ has a representation (3.1) with

$$
\alpha_{r}(d, \delta)=\frac{1}{r^{2}} \sum_{D|r ; E| r} F_{r}\left(\left(\frac{r}{D}, \frac{r}{E}\right)\right) c_{D}\left(\frac{r}{d}\right) c_{E}\left(\frac{r}{\delta}\right) .
$$

Rewriting and applying (2.14), one obtains

$$
\begin{aligned}
\alpha_{r}(d, \delta) & =\frac{1}{r^{2}} \sum_{\Delta \mid r} F_{r}(\Delta) \sum_{D|r, E| r ;(r / D, r / E)=\Delta} c_{D}\left(\frac{r}{d}\right) c_{E}\left(\frac{r}{\delta}\right) \\
& =\frac{1}{r^{2}} \sum_{\Delta \mid r} F_{r}(\Delta) \sum_{D|r, E| r ;[D, E]=r / \Delta} c_{D}\left(\frac{r}{d}\right) c_{E}\left(\frac{r}{\delta}\right) .
\end{aligned}
$$

Hence by Lemma 4, we have

$$
\alpha_{r}(d, \delta)=\frac{1}{r^{2}} \sum_{\Delta \mid r} F_{r}(\Delta) c r / \Delta\left(\frac{r}{d}, \frac{r}{\delta}\right)=\frac{1}{r^{2}} \sum_{\Delta \mid r} F_{r}\left(\frac{r}{\Delta}\right) c_{\Delta}\left(\frac{r}{d}, \frac{r}{\Delta}\right) .
$$

Therefore, since $c_{r}(m, n)=C_{r}((m, n))$, we have by definition of $\beta_{r}(d), \alpha_{r}(d, \delta)$ $=\beta_{r}((r / d, r / \delta))$. By (3.1) it follows then that

$$
f_{r}(m, n)=\frac{1}{r^{2}} \sum_{d|r ; \delta| r} \beta_{r}\left(\left(\frac{r}{d}, \frac{r}{\delta}\right)\right) c_{d}(m) c_{\delta}(n) .
$$

We may apply to (4.10) the same argument as that used in passing from (4.8) to (4.9), thereby obtaining

$$
f_{r}(m, n)=\frac{1}{r^{2}} \sum_{d \mid r} \beta_{r}\left(\frac{r}{d}\right) c_{d}(m, n) .
$$

Since $\beta_{r}(r / d)=\alpha_{r}(d)$, we have proved (4.5), (4.6). The uniqueness of the coefficients $\alpha_{r}(d)$ follows from the uniqueness of the $\alpha_{r}(d, \delta)$ in Theorem 1.

We now prove the equivalence of (4.6) and (4.7). Denote the right member of (4.7) by $\alpha_{r}^{\prime}(d)$. Then

$$
\alpha_{r}^{\prime}(d)=\frac{1}{r^{2} J^{2}(d)} \sum_{a, b(\bmod r)} F_{r}((a, b)) c_{d}(a, b) .
$$

Placing $\{a, b\}=r / D\{x, y\},((x, y), D)=1$, we obtain $\left(^{*}\right)$ by Remark 2, since $F_{r}(n)$ is even $(\bmod r)$,

$$
\begin{aligned}
\alpha_{r}^{\prime}(d) & =\frac{1}{r^{2} J(d)} \sum_{D \mid r ;(x, y, D)=1} F_{r}\left(\left(\frac{r x}{D}, \frac{r y}{D}\right)\right) c_{d}\left(\frac{r x}{D}, \frac{r y}{D}\right) \\
& =\frac{1}{r^{2} J(d)} \sum_{D \mid r} F_{r}\left(\frac{r}{D}\right) J(D) C_{d}\left(\frac{r}{D}\right) .
\end{aligned}
$$


By Lemma 3, it follows then that $\alpha_{r}^{\prime}(d)=\alpha_{r}(d)$.

The converse follows by observing that, for each divisor $d$ of $r, c_{d}(m, n)$ $=C_{d}((m, n))=C_{d}((m, n, r))$, and hence that $c_{d}(m, n)$ is totally even $(\bmod r)$. This completes the proof of Theorem 2 .

Analogous to Corollary 1.1 we have the following inversion relation for totally even functions $(\bmod r)$.

CoROllaRy 2.1. If $f_{r}(m, n)=F_{r}((m, n))$ is a totally even function $(\bmod r)$ defined by

$$
f_{r}(m, n)=\sum_{d \mid r} g_{r}(d) c_{d}(m, n)
$$

then

$$
g_{r}\left(\frac{r}{(m, n, r)}\right)=\frac{1}{r^{2}} \sum_{d \mid r} F_{r}\left(\frac{r}{d}\right) c_{d}(m, n) .
$$

Proof. In (4.5) and (4.6), replace $\alpha_{r}(d)$ by $g_{r}(d)$, and in (4.6) place $d=r /(m, n, r)$.

Finally we deduce from Theorem 2 the following arithmetical representation.

Corollary 2.2. If $f_{r}(m, n)$ is defined by (4.5), then

$$
f_{r}(m, n)=\sum_{d \mid(m, n, r)} d^{2} \sum_{\delta \mid r / d} \alpha_{r}(d \delta) \mu(\delta) .
$$

Proof. Applying (4.1) to (4.5) one obtains

$$
f_{r}(m, n)=\sum_{d \mid r} \alpha(d) \sum_{D \mid(m, n, d) ;(d=D E)} D^{2} \mu\left(\frac{d}{D}\right)=\sum_{D \mid(m, n, r)} D^{2} \sum_{E \mid r / D} \alpha_{r}(D E) \mu(E) .
$$

5. Applications to simultaneous congruences. In this section we apply Theorem 1 to obtain simple expansions for the number of solutions of certain types of simultaneous pairs of congruences. We remark that the alternative formula (3.3) for the Fourier coefficients is particularly suited for such applications.

The first three applications will be to congruences of the form,

$$
\begin{aligned}
m & \equiv x_{1} y_{1}+\cdots+x_{8} y_{s}(\bmod r), \\
n & \equiv x_{1}+\cdots+x_{s} \quad(\bmod r) .
\end{aligned}
$$

THEOREM 3. The number of solutions $P_{r, s}(m, n)$ of $(5.1)$ in $x_{i}, y_{i}(\bmod r)$, $i=1, \cdots, s$, is given by

$$
P_{r, s}(m, n)=r^{2 \sigma-2} \sum_{d|r ; \delta| d} \frac{c(m, d) c(n, \delta)}{d^{s}} .
$$


Proof. Clearly $P_{r, s}(m, n)$ is even $(\bmod r)$. With $P_{r, s}(m, n)=f_{r}(m, n)$, it follows that $P_{r, s}(m, n)$ is given by (3.1), where

$$
\begin{aligned}
\alpha_{r}(d, \delta) & =\frac{1}{r^{2} \phi(d) \phi(\delta)} \sum_{x_{i}, y_{i}(\bmod r) ;(i=1, \ldots, s)} c_{d}\left(x_{1} y_{1}+\cdots+x_{s} y_{s}\right) c_{\delta}\left(x_{1}+\cdots+x_{s}\right) \\
& =\frac{1}{r^{2} \phi(d) \phi(\delta)} \sum_{(u, d)=1 ;(v, \delta)=1}\left(\sum_{x(\bmod r)} e_{\delta}(v x) \sum_{y(\bmod r)} e_{d}(u x y)\right)^{s} \\
& =\frac{r^{8-2}}{d^{s} \phi(d) \phi(\delta)} \sum_{(u, d)=1_{i}(v, \delta)=1}\left(\sum_{x(\bmod r)} e_{\delta}(v x) \eta_{d}(u x)\right)^{s} .
\end{aligned}
$$

By (2.2), $\eta_{d}(u x)=0$ unless $d \mid x$; therefore, we may replace $x$ by $x=d X$, $X(\bmod r / d)$, to obtain

$$
\alpha_{r}(d, \delta)=\frac{r^{s-2}}{\phi(\delta)} \sum_{(v, \delta)=1}\left(\sum_{X(\bmod r / d)} e_{\delta}(v d X)\right)^{s}=\frac{r^{s-2}}{\phi(\delta)} \sum_{(v, \delta)=1}\left(\eta_{r / d}\left(\frac{v r}{\delta}\right)\right)^{s} .
$$

Hence, again $\left(^{*}\right)$ by $(2.2)$,

$$
\alpha_{r}(d, \delta)= \begin{cases}\frac{r^{2 s-2}}{d^{s}} & (\delta \mid d), \\ 0 & (\delta \nmid d) .\end{cases}
$$

The theorem follows from (3.1) and (5.3).

THEOREM 4. The number of solutions $P_{r, s}^{\prime}(m, n)$ of $(5.1)$ in $x_{i}, y_{i}(\bmod r)$, such that $\left(y_{i}, r\right)=1, i=1, \cdots, s$, is given by

$$
P_{r, s}^{\prime}(m, n)=\frac{\phi^{s}(r)}{r^{2-s}} \sum_{d \mid r} \frac{c_{d}(m) c_{d}(n)}{\phi^{s}(d)} .
$$

Proof. It is easily observed that $P_{r, s}^{\prime}(m, n)$ is an even function of $m, n(\bmod r)$. Hence, as in the previous proof, we place $P_{r, s}^{\prime}(m, n)=f_{r}(m, n)$ in (3.1), so that by (3.3) and Remark 1,

$$
\begin{aligned}
\alpha_{r}(d, \delta) & =\frac{1}{r^{2} \phi(d) \phi(\delta)} \sum_{x_{i}, y_{i}(\bmod r) ;\left(y_{i}, r\right)=1 ; i=1, \cdots, s} \cdot c_{d}\left(x_{1} y_{1}+\cdots+x_{s} y_{s}\right) c_{\delta}\left(x_{1}+\cdots+x_{s}\right) \\
& =\frac{1}{r^{2} \phi(d) \phi(\delta)} \sum_{(u, d)=1 ;(v, \delta)=1}\left(\sum_{x(\bmod r)} e_{\delta}(v x) \sum_{(y, r)=1} e_{d}(u x y)\right)^{s} \\
& =\frac{\phi^{s}(r)}{r^{2} \phi^{s}(d) \phi(\delta)} \sum_{(v, \delta)=1}\left(\sum_{x(\bmod r)} e_{\delta}(v x) c_{d}(x)\right)^{s} .
\end{aligned}
$$

Replacing $x$ by $(r / D) X,(X, D)=1$, one obtains 
1960]

$$
\begin{aligned}
\alpha_{r}(d, \delta) & =\frac{\phi^{s}(r)}{r^{2} \phi^{s}(d) \phi(\delta)} \sum_{(v, \delta)=1}\left(\sum_{D \mid r ;}(X, D)=1\right. \\
& =\frac{\phi^{s}(r)}{r^{2} \phi^{s}(d)}\left(\sum_{D \mid r} c_{d}\left(\frac{r}{D}\right) c_{D}\left(\frac{r}{D}\right)\right)^{s} .
\end{aligned}
$$

By (2.7), one obtains then

$$
\alpha_{r}(d, \delta)= \begin{cases}r^{s-2}\left(\frac{\phi(r)}{\phi(d)}\right)^{s} & \text { if } d=\delta, \\ 0 & \text { if } d \neq \delta .\end{cases}
$$

The theorem follows by (3.1) and (5.5).

The case $s=1$ of Theorem 4 yields

Corollary 4.1.

$$
\sum_{d \mid r} \frac{c_{d}(m) c_{d}(n)}{\phi(d)}=\left\{\begin{array}{cc}
\frac{r}{\phi(r /(n, r))} & \text { if }(m, r)=(n, r), \\
0 & \text { if }(m, r) \neq(n, r) .
\end{array}\right.
$$

THEOREM 5. The number of solutions $P_{r, s}^{*}(m, n)$ of $(5.1)$ in $x_{i}, y_{i}(\bmod r)$, $i=1, \cdots$, such that $\left(\left(y_{i}\right), r\right) \equiv\left(\left(y_{1}, \cdots, y_{s}\right), r\right)=1$, is given by

$$
P_{r, 8}^{*}(m, n)=\frac{J_{s}(r)}{r^{2-s}} \sum_{d \mid r} \frac{c_{d}(m) c_{d}(n)}{J_{s}(d)} .
$$

Proof. Since $P_{r, s}^{*}(m, n)$ is even $(\bmod r)$ we put $P_{r, s}^{*}(m, n)=f_{r}(m, n)$ in (3.1), where, by (3.3),

$$
\begin{aligned}
& \alpha_{r}(d, \delta)=\frac{1}{r^{2} \phi(d) \phi(\delta)} \sum_{x_{i}, y_{i}(\bmod r) ; i=1, \cdots, z ;\left(\left(y_{i}\right), r\right)=1} \\
&=\frac{1}{\cdot_{d}\left(x_{1} y_{1}+\cdots+x_{s} y_{s}\right) c_{\delta}\left(x_{1}+\cdots+x_{s}\right)} \\
& \sum_{r^{2} \phi(d) \phi(\delta)}\left(\prod_{(u, d)=1 ;(v, \delta)=1}^{\delta} \sum_{i=1} e_{x_{i}, y_{i}(\bmod r) ;\left(\left(y_{i}\right), r\right)=1} e_{d}\left(u x_{i} y_{i}\right) e_{\delta}\left(v x_{i}\right)\right) .
\end{aligned}
$$

By Remark 2, we place $x_{i}=\left(r / D_{i}\right) X_{i},\left(X_{i}, D_{i}\right)=1, i=1, \cdots, s$, to obtain $\alpha_{r}(d, \delta)$

$$
\begin{array}{r}
=\frac{1}{r^{2} \phi(d) \phi(\delta)} \sum_{(u, d)=1 ;(v, \delta)=1}\left(\prod_{i=1}^{\dot{1}} \sum_{D_{i} \mid r ; y_{i}(\bmod r) ;\left(\left(y_{i}\right), r\right)=1} \sum_{\left(X_{i}, D_{i}\right)=1}\right. \\
\cdot e_{D_{i}}\left(\frac{u r X_{i} y_{i}}{d}\right) e_{D_{i}}\left(\frac{v r X_{i}}{\delta}\right) \\
=\frac{1}{r^{2} \phi(d) \phi(\delta)} \sum_{(u, d)=1 ;(v, \delta)=1}\left(\prod_{i=1}^{\dot{j}} \sum_{y_{i}(\bmod r) ;\left(\left(y_{i}\right), r\right)=1} \sum_{D_{i} \mid r} c_{D_{i}}\left(\frac{u r y_{i}}{d}+\frac{v r}{\delta}\right)\right) .
\end{array}
$$


By (2.8) the product is $\neq 0$ if and only if $r\left(u y_{i} \delta+v d\right) / d \delta \equiv 0(\bmod r), i=1$, $\cdots, s$; that is, if and only if $u y_{i} \delta+v d \equiv 0(\bmod d \delta), i=1, \cdots, s$. But the latter relation would imply that $\delta \mid d, d=D \delta$, so that $u y_{i}+v D \equiv 0(\bmod d), i$ $=1, \cdots, s$, which would in turn imply that $D=1$, since $(u, d)=\left(\left(y_{i}\right), d\right)=1$. Hence a necessary and sufficient condition that the product be $\neq 0$ is that $d=\delta, u y_{i}+v \equiv 0(\bmod d), i=1, \cdots, s$. Thus it follows by (2.8) that $\alpha_{r}(d, \delta)$ $=0$ if $d \neq \delta$ and that

$$
\alpha_{r}(d, d)=\frac{r^{8-2}}{\phi^{2}(d)} \sum_{(u, d)=1 ;(v, d)=1}\left(\sum_{y_{i} \equiv-v u^{-1}(\bmod d) ; i=1, \ldots, s ;\left(\left(y_{i}\right), r\right)=1} 1\right) .
$$

By Remark 1, we obtain then

$$
\alpha_{r}(d, \delta)= \begin{cases}r^{s-2}\left(\frac{J_{s}(r)}{J_{s}(d)}\right) & \text { if } d=\delta, \\ 0 & \text { if } d \neq \delta .\end{cases}
$$

(5.6) follows from (3.1) and (5.7).

We next obtain formulas for the number of solutions $(\bmod r)$ of a simultaneous pair of bilinear congruences,

$$
\begin{aligned}
m & \equiv x_{1} y_{1}+\cdots+x_{8} y_{8}(\bmod r), \\
n & \equiv x_{1} z_{1}+\cdots+x_{8} z_{8}(\bmod r) .
\end{aligned}
$$

THEOREM 6. The number of solutions $Q_{r, 8}(m, n)$ of (5.8) in $x_{i}, y_{i}, z_{i}(\bmod r)$, $i=1, \cdots, s$, is given by

$$
Q_{r, s}(m, n)=r^{3 s-2} \sum_{d|r ; \delta| r} \frac{c_{d}(m) c_{\delta}(n)}{\left[d, \delta \bar{j}^{s}\right.},
$$

or by the equivalent formula,

$$
Q_{r, s}(m, n)=r^{38-2} \sum_{d \mid r} \frac{c_{d}(m, n)}{d^{s}} .
$$

Proof. Certainly $Q_{r, s}(m, n)$ is even $(\bmod r)$. With $f_{r}(m, n)=Q_{r, s}(m, n)$ in (3.1) we have by (3.3),

$$
\begin{aligned}
\alpha_{r}(d, \delta)= & \frac{1}{r^{2} \phi(d) \phi(\delta)} \\
& \cdot \sum_{x_{i}, y_{i}, z_{i}(\bmod r) ;(i=1, \ldots, s)} c_{d}\left(x_{1} y_{1}+\cdots+x_{s} y_{\delta}\right) c_{\delta}\left(x_{1} z_{1}+\cdots+x_{z} z_{8}\right) \\
& =\frac{1}{r^{2} \phi(d) \phi(\delta)} \sum_{(u, d)=1 ;(v, \delta)=1}\left(\sum_{x, z(\bmod r)} e_{\delta}(v x z) \sum_{y(\bmod r)} e_{d}(u x y)\right)^{s} \\
& =\frac{r^{2 s-2}}{\delta^{s} d^{s} \phi(d) \phi(\delta)} \sum_{(u, d)=1 ;(v, \delta)=1}\left(\sum_{x(\bmod r) ; z(\bmod \delta)} e_{\delta}(v x z) \eta_{d}(u x)\right)^{s} .
\end{aligned}
$$


Now $\eta_{d}(u x)=0$ unless $d \mid x$. Placing therefore $x=d X, X(\bmod r / d)$, we obtain by $(2.2)$,

$$
\alpha_{r}(d, \delta)=\frac{r^{28-2}}{\delta^{s} \phi(\delta)} \sum_{(v, \delta)=1}\left(\sum_{X(\bmod r / d)} \eta_{\delta}(v d X)\right)^{s} .
$$

But $\eta_{\delta}(v d X)=0$ unless $\delta \mid d X$, that is, unless $\delta /(d, \delta)$ divides $X$. Since $\delta d \mid r(d, \delta)$, we have

$$
\alpha_{r}(d, \delta)=r^{2 s-2}\left(\sum_{X(\bmod r / d) ; \delta /(d, \delta) \mid X} 1\right)^{s}=r^{2 s-2}\left(\frac{r / d}{\delta /(d, \delta)}\right)^{s},
$$

and hence by (2.15),

$$
\alpha_{r}(d, \delta)=\frac{r^{3 s-2}}{[d, \delta]^{8}} .
$$

(5.9) results from (3.1) and (5.11). But (5.9) can be rewritten in the form (5.10) on the basis of Lemma 4. This completes the proof of the theorem.

We now deduce a simple arithmetical representation of $Q_{r, s}(m, n)$ from (5.10). First it is noted, by (2.1), that

THEOREM $6^{\prime}$.

$$
Q_{r, s}(m, n)=r^{2 s-2} \sum_{d \mid(m, n, r)} d^{2} J_{s}\left(\frac{r}{d}\right) .
$$

Proof. By (5.10), (4.5), and Corollary 2.2, one obtains with $\alpha_{r}(d)=r^{3 s-2} / d^{s}$,

$$
Q_{r, s}(m, n)=r^{3 s-2} \sum_{d \mid(m, n, r)} d^{2-s} \sum_{\delta \mid r / d} \frac{\mu(\delta)}{\delta^{s}}
$$

and (5.13) follows by virtue of (2.1).

By either (5.10) or (5.13) it follows that

Corollary 6.1. The function $Q_{r, 8}(m, n)$ is a totally even function of $m, n(\bmod r)$.

6. Simultaneous restricted partitions $(\bmod r)$. In this section we determine the number of solutions of simultaneous pairs of linear congruences with various types or restrictions on the summands. The first application (Theorem 7) is based on a direct proof in the manner of the preceding section. The remaining results are obtained using Cauchy products $(\bmod r)$.

We consider the congruence pair,

$$
\begin{aligned}
m & \equiv x_{1}+\cdots+x_{8}(\bmod r), \\
n & \equiv y_{1}+\cdots+y_{8}(\bmod r) .
\end{aligned}
$$

THEOREM 7. The number of solutions $G_{r, 8}(m, n)$ of $(6.1)$ in $x_{i}, y_{i}(\bmod r)$, $i=1, \cdots, s$, such that $\left(\left(x_{i}, y_{i}\right), r\right) \equiv\left(x_{1}, y_{1}, \cdots, x_{s}, y_{s}, r\right)=1$, is given by 


$$
G_{r, s}(m, n)=\frac{J_{2 s}(r)}{r^{2}} \sum_{d \mid r}\left(\frac{\mu(d)}{J_{2 s}(d)}\right) c_{d}(m, n) .
$$

Note. By (2.11), $G_{r, s}(m, n)$ has the equivalent form

$$
G_{r, \&}(m, n)=\frac{1}{r^{2}} \sum_{d \mid r} c_{r}^{(2 s)}\left(\frac{r}{d}\right) c_{d}(m, n) .
$$

Proof. Since $G_{r, 8}(m, n)$ is obviously even $(\bmod r)$, we place, as in $\S 5$, $f_{r}(m, n)=G_{r, s}(m, n)$ in (3.1), where

$$
\begin{aligned}
& \alpha_{r}(d, \delta)=\frac{1}{r^{2} \phi(d) \phi(\delta)} \sum_{x_{i}, y_{i}(\bmod r)_{i}\left(\left(x_{i}, y_{i}\right), r\right)=1} c_{d}\left(x_{1}+\cdots+x_{s}\right) c_{\delta}\left(y_{1}+\cdots+y_{8}\right) \\
& =\frac{1}{r^{2} \phi(d) \phi(\delta)} \sum_{(u, d)=1 ;} \sum_{(v, \delta)=1} \sum_{x_{i}, y(\bmod r) ;\left(\left(x_{i}, y_{i}\right), r\right)=1} \\
& \cdot e_{d}\left(u\left(x_{1}+\cdots+x_{s}\right) e_{\delta}\left(v\left(y_{1}+\cdots+y_{8}\right)\right)\right) \text {. }
\end{aligned}
$$

We place now $[d, \delta]=\Delta$ and define $d D=\delta E=\Delta$. One therefore obtains

$$
\begin{aligned}
& \alpha_{r}(d, \delta)=\frac{1}{r^{2} \phi(d) \phi(\delta)} \sum_{(u, d)=1 ;(v, \delta)=1} \sum_{x_{i}, y_{i}(\bmod r) ;\left(\left(x_{i}, y_{i}\right), r\right)=1} \\
& \cdot e_{\Delta}\left(D u\left(x_{1}+\cdots+x_{s}\right)+E v\left(y_{1}+\cdots+y_{s}\right)\right) .
\end{aligned}
$$

Hence by Remark 1 and the definition (2.12), it follows that $\alpha_{r}(d, \delta)=\frac{1}{r^{2} \phi(d) \phi(\delta)}\left(\frac{J_{2 s}(r)}{J_{2 s}(\Delta)}\right) \sum_{(u, d)=1 ;(v, \delta)=1} c_{\Delta}(D u, \cdots, D U, E v, \cdots, E v)$, where the arguments $D u$ and $E v$ each occur $s$ times. Now $(D, E)=1$, so that on applying Lemma $2\left(^{*}\right)$,

$$
\alpha_{r}(d, \delta)=\frac{J_{2 s}(r)}{r^{2} J_{2 s}(\Delta)} c_{\Delta}^{(2 s)}(1)=\frac{J_{2 s}(r)}{r^{2}}\left(\frac{\mu(\Delta)}{J_{2 s}(\Delta)}\right) .
$$

By (3.1) and (6.3) we deduce then

$$
G_{r, s}(m, n)=\frac{J_{2 s}(r)}{r^{2}} \sum_{\Delta \mid r ;[d, \delta]=\Delta}\left(\frac{\mu(\Delta)}{J_{2 s}(\Delta)}\right) c_{d}(m) c_{\delta}(n),
$$

and (6.2) results on applying Lemma 4.

Corollary 7.1. The function $G_{r, s}(m, n)$ is totally even $(\bmod r)$.

The remaining portion of this section is based on the following orthogonality property of the Ramanujan sums $[4,(1.2)]$,

LEMma 5. If $d$ and $\delta$ are divisors of $r$, then 


$$
S_{d, \delta}(n) \equiv \sum_{n \equiv a+b(\bmod r)} c_{d}(a) c_{\delta}(b)= \begin{cases}r c_{d}(n) & \text { if } d=\delta, \\ 0 & \text { if } d \neq \delta .\end{cases}
$$

We prove the following analogue of Lemma 5 .

Lemma 6. If $d$ and $\delta$ are divisors of $r$, then

$$
\begin{aligned}
S_{d, \delta}(m, n) & \equiv \sum_{m \equiv a+b(\bmod r) ; n \equiv a^{\prime}+b^{\prime}(\bmod r)} c_{d}\left(a, a^{\prime}\right) c_{\delta}\left(b, b^{\prime}\right) \\
& = \begin{cases}r^{2} c_{d}(m, n) & \text { if } d=\delta, \\
0 & \text { if } d \neq \delta .\end{cases}
\end{aligned}
$$

Proof. By Lemma 4, one obtains

$$
S_{d, \delta}(m, n)=\sum_{[D, E]=d_{i}\left[D^{\prime}, E^{\prime}\right]=\delta}\left(\sum_{m \equiv a+b(\bmod r)} c_{D}(a) c_{D^{\prime}}(b) \sum_{n \equiv a^{\prime}+b^{\prime}(\bmod r)} c_{E}\left(a^{\prime}\right) c_{E^{\prime}}\left(b^{\prime}\right)\right) .
$$

But by (6.5), the inner expression is 0 if $D \neq D^{\prime}$ or $E \neq E^{\prime}$. In particular, $S_{d, \delta}(m, n)=0$ if $d \neq \delta$, thus proving one part of (6.6). In the remaining case, $d=\delta$, we may therefore limit the summation to values $D=D^{\prime}, E=E^{\prime}$, obtaining, by Lemmas 5 and 4 ,

$$
S_{d, d}(m, n)=r^{2} \sum_{[D, E]=d} c_{D}(m) c_{E}(n)=r^{2} c_{d}(m, n) .
$$

This completes the proof.

THEOREM 8. The number of solutions $H_{r, s}(m, n)$ of $(6.1)$ in $x_{i}, y_{i}(\bmod r)$, such that $\left(x_{i}, y_{i}, r\right)=1, i=1, \cdots, s$, is given by

$$
H_{r, s}(m, n)=\frac{1}{r^{2}} \sum_{d \mid r} C_{r}^{s}\left(\frac{r}{d}\right) c_{d}(m, n) .
$$

REMARK 5. By (4.2), we may restate (6.7) in the equivalent form,

$$
H_{r, s}(m, n)=\frac{J^{s}(r)}{r^{2}} \sum_{d \mid r}\left(\frac{\mu(d)}{J(d)}\right)^{s} c_{d}(m, n) .
$$

Proof. Case $1(s=1)$. Define $\rho_{r}(n)$ to be 1 or 0 according as $(n, r)=1$ or $\neq 1$, and place $\rho_{r}(m, n)=\rho_{r}((m, n))$, so that $H_{r, 1}(m, n)=\rho_{r}(m, n)$. Evidently $\rho_{r}(m, n)$ is a totally even function of $m, n(\bmod r)$. We give two proofs to illustrate different methods.

First proof $(s=1)$. Let $f_{r}(m, n)=\rho_{r}(m, n)$ in (4.5), so that by (4.7), Remark 1, and Lemma 2,

$$
\alpha_{r}(d)=\frac{1}{r^{2} J(d)} \sum_{(x, y, r)=1} c_{d}(x, y)=\frac{J(r)}{r^{2} J^{2}(d)} \sum_{(x, y, d)=1} C_{d}(1)=\frac{J(r)}{r^{2}}\left(\frac{\mu(d)}{J(d)}\right) .
$$


By (4.5) we therefore obtain (6.8) with $s=1$.

Second proof $(s=1)$. Clearly

$$
C_{r}((m, n))=c_{r}(m, n)=\sum_{d \mid r} \rho_{r}\left(\frac{r}{d}\right) c_{d}(m, n) .
$$

Hence by Corollary 2.1, with $g_{r}(d)=\rho_{r}(r / d)$, we have

$$
\rho_{r}((m, n, r))=\rho_{r}(m, n)=\frac{1}{r^{2}} \sum_{d \mid r} C_{r}\left(\frac{r}{d}\right) c_{d}(m, n) .
$$

This proves (6.7) in case $s=1$.

CASE $2(s>1)$. We proceed by induction, assuming the theorem, in the form (6.7), for arbitrary $s=t>1$. Then

$$
H_{r, t+1}(m, n)=\sum_{m \equiv a+b(\bmod r) ; n \equiv a^{\prime}+b^{\prime}(\bmod r)} H_{r, t}\left(a, a^{\prime}\right) H_{r, 1}\left(b, b^{\prime}\right),
$$

so that by the induction hypothesis and the theorem for $s=1$, we obtain, using the notation of Lemma 6,

$$
H_{r, t+1}(m, n)=\frac{1}{r^{4}} \sum_{d|r ; \delta| r} C_{r}^{t}\left(\frac{r}{d}\right) C_{r}\left(\frac{r}{\delta}\right) S_{d, \delta}(m, n) .
$$

(6.7) follows immediately in the case $s=t+1$, by virtue of (6.6). The induction is complete.

REMARK 6. The proof of Theorem 8 illustrates the following principle, which is a consequence of Theorem 2 and Lemma 6: The Cauchy product of two totally even functions $(\bmod r)$ is also totally even $(\bmod r)$.

In the remainder we consider mixed congruence pairs, $s \geqq 1, t \geqq 1$,

$$
\begin{aligned}
m & \equiv u_{1}+\cdots+u_{s}+x_{1}+\cdots+x_{t}(\bmod r), \\
n & \equiv v_{1}+\cdots+v_{s}+y_{1}+\cdots+y_{t}(\bmod r) .
\end{aligned}
$$

In particular we have

THEOREM 9. The number of solutions $L_{r}^{s, t}(m, n)$ of $(6.10)$ in $u_{i}, v_{i}, x_{j}$, $y_{j}(\bmod r)$, such that $\left(u_{1}, v_{1}, \cdots, u_{s}, v_{s}, r\right)=1,\left(x_{j}, y_{j}, r\right)=1, i=1, \cdots, s ; j$ $=1, \cdots, t$, is given by

$$
L_{r}^{s, t}(m, n)=\frac{J_{2 s}(r) J^{t}(r)}{r^{2}} \sum_{d \mid r}\left(\frac{\mu^{t+1}(d)}{J_{2 s}(d) J^{t}(d)}\right) c_{d}(m, n) .
$$

Proof. This result follows from the fact that $L_{r}^{s, t}(m, n)$ is the Cauchy product $(\bmod r)$ of $G_{r, s}(m, n)$ and $H_{r, t}(m, n)$, in conjunction with Lemma 6, and Theorems 7 and 8.

We shall need the following result proved in [1, Theorem 7], (cf. also $[6$, Theorem 7; 7, Theorem 7]). 
Lemma 7. The number of solutions in $x_{i}(\bmod r),\left(x_{i}, r\right)=1, i=1, \cdots, s$, of the congruence $n \equiv x_{1}+\cdots+x_{s}(\bmod r)$, is given by

$$
\phi_{r}^{(s)}(n)=\frac{\phi^{s}(r)}{r} \sum_{d \mid r}\left(\frac{\mu(d)}{\phi(d)}\right)^{s} c_{d}(n) .
$$

Place $\phi_{r, s}(m, n)=\phi_{r}^{(s)}(m) \phi_{r}^{(s)}(n)$. Further let $M_{r}^{s, t}(m, n)$ represent the number of solutions of $(6.10)$ in $u_{i}, v_{i}, x_{j}, y_{j}(\bmod r)$ such that $\left(u_{i}, r\right)=\left(v_{i}, r\right)$ $=1,\left(x_{1}, y_{1}, \cdots, x_{t}, y_{t}, r\right)=1, i=1, \cdots, s ; j=1, \cdots, t$; similarly, let $N_{r}^{s, t}(m, n)$ denote the number of solutions under the conditions, $\left(u_{i}, r\right)$ $=\left(v_{i}, r\right)=1,\left(x_{j}, y_{j}, r\right)=1$. It is clear that $M_{r}^{s, t}(m, n)$ is the Cauchy product $(\bmod r)$ of $\phi_{r, s}(m, n)$ by $G_{r, t}(m, n)$, while $N_{r}^{s, t}(m, n)$ is the Cauchy product $(\bmod r)$ of $\phi_{r, s}(m, n)$ by $H_{r, t}(m, n)$. We denote these facts symbolically by writing $M_{r}^{s, t}=\phi_{r, s} \cdot G_{r, \ell}, N_{r}^{s, l}=\phi_{r, s} \cdot H_{r, t}$.

TheOREM 10.

$$
M_{r}^{8, t}(m, n)=\frac{\phi^{2 s}(r) J_{2 t}(r)}{r^{2}} \sum_{\Delta \mid r ;[d, \delta]=\Delta}\left(\frac{\mu(d) \mu(\delta)}{\phi(d) \phi(\delta)}\right)^{\prime}\left(\frac{\mu(\Delta)}{J_{2 t}(\Delta)}\right) c_{d}(m) c_{\delta}(n) .
$$

Proof. Since $M_{r}^{s, t}=\phi_{r, s} \cdot G_{r, t}$, we have, by Lemma 7 and (6.4), using the notation of Lemma 5 ,

$$
\begin{aligned}
& M_{r}^{s, t}(m, n) \\
& \quad=\frac{\phi^{2 \delta}(r) J_{2 t}(r)}{r^{4}} \sum_{d|r ; \delta| r}\left(\frac{\mu(d) \mu(\delta)}{\phi(d) \phi(\delta)}\right)^{s} \sum_{\Delta \mid r ;\left[d^{\prime}, \delta^{\prime}\right]=\Delta}\left(\frac{\mu(\Delta)}{J_{2 t}(\Delta)}\right) S_{d, d^{\prime}}(m) S_{\delta, \delta^{\prime}}(n) ;
\end{aligned}
$$

consequently, (6.13) results on applying (6.5).

A similar approach leads to the following representation of $N_{r}^{s, t}(m, n)$.

THEOREM 11.

$$
N_{r}^{s, t}(m, n)=\frac{\phi^{2 s}(r) J^{t}(r)}{r^{2}} \sum_{\Delta \mid r_{i}[d, \delta]=\Delta}\left(\frac{\mu(d) \mu(\delta)}{\phi(d) \phi(\delta)}\right)^{s}\left(\frac{\mu(\Delta)}{J(\Delta)}\right)^{t} c_{d}(m) c_{\delta}(n) .
$$

Finally, we return to the question of arithmetical representations, treating, as an illustration, the function $G_{r, s}(m, n)$. We first note two further properties of $J_{k}(r),[3,(7.8)]$,

$$
\begin{aligned}
J_{k}\left(r_{1} r_{2}\right) & =J_{k}\left(r_{1}\right) J_{k}\left(r_{2}\right) \frac{\delta^{k}}{J_{k}(\delta)}, & & \left(\delta=\left(r_{1}, r_{2}\right)\right) \\
\sum_{d \mid r ;(d, n)=1} \frac{\mu(d)}{J_{k}(d)} & =\left(\frac{r}{\delta}\right)^{k} \frac{J_{k}(\delta)}{J_{k}(r)}, & & (\delta=(n, r)) .
\end{aligned}
$$

Property (6.15) reduces to the ordinary factorability property of $J_{k}(r)$ when $\left(r_{1}, r_{2}\right)=1$. 
TheOREM $7^{\prime}$.

$$
G_{r, 8}(m, n)=\left(\frac{r}{(m, n, r)}\right)^{2 s-2} J_{2 s-2}((m, n, r)) .
$$

Proof. By (6.2), (4.5), and (4.13), with $\alpha_{r}(d)=J_{2 s}(r) \mu(d) / r^{2} J_{2 s}(d)$, it follows, using the factorability of $\mu(r)$ and $J_{k}(r)$, that

$$
r^{2} G_{r, s}(m, n) / J_{2 s}(r)=\sum_{d \mid(m, n, r)} d^{2} \sum_{\delta \mid r / d} \frac{\mu(\delta d) \mu(\delta)}{J_{2 s}(d \delta)}=\sum_{d \mid(m, n, r)} \frac{d^{2} \mu(d)}{J_{2 \delta}(d)} \sum_{\delta \mid r / d ;(\delta, d)=1} \frac{\mu^{2}(\delta)}{J_{28}(\delta)} .
$$

Hence, by (6.16) and (6.15), one obtains

$$
\begin{aligned}
G_{r, s}(m, n) & =\frac{J_{2 s}(r)}{r^{2}} \sum_{d \mid(m, n, r) ; d e=r} \frac{d^{2} \mu(d)}{J_{2 s}(d)}\left(\frac{e}{(d, e)}\right)^{2 s} \frac{J_{2 s}((d, e))}{J_{2 s}(e)} \\
& =r^{2 s-2} \sum_{d \mid(m, n, r)} \frac{\mu(d)}{d^{2 s-2}}
\end{aligned}
$$

and (6.17) follows on the basis of (2.1).

7. Primitive functions $(\bmod r)$. In $[3, \S 7]$ it was proved that a function $f_{r}(n)$ is primitive $(\bmod r)$ if and only if $f_{r}(n)$ has a Fourier representation of the form,

$$
f_{r}(n)=\sum_{d \mid \gamma(r)} \alpha_{r}(d) c_{d}(n) .
$$

We prove an analogous result for even functions of two arguments $(\bmod r)$.

TheOREM 12. A function $f_{r}(m, n)$ is a primitive function of $m$ and $n(\bmod r)$ if and only if its Fourier representation is of the form

$$
f_{r}(m, n)=\sum_{d|\gamma(r) ; \delta| \gamma(r)} \alpha_{r}(d, \delta) c_{d}(m) c_{\delta}(n) .
$$

Proof. Clearly any function $(7.1)$ is primitive $(\bmod r)$. Conversely, suppose that $f(n, r)$ is primitive $(\bmod r)$ with Fourier representation (3.1). As a function of the argument $m$ alone, the expansion of $f_{r}(m, n)$ may therefore be written

$$
f_{r}(m, n)=\sum_{d \mid r} \beta_{r}(n, d) c(m, d)
$$

where, for all $n$,

$$
\beta_{r}(n, d)=\sum_{\delta \mid r} \alpha_{r}(d, \delta) c(n, \delta) .
$$

But by the result of [3] cited above, since $f_{r}(m, n)$ is primitive $(\bmod r)$ as a function of $m$, it must follow, for each nonprimitive $d(d \nmid \gamma(r))$, that $\beta_{r}(n, d)=0$. By the uniqueness [1] of the $\alpha_{r}(d, \delta)$ in (7.3), this implies that 
$\alpha_{r}(d, \delta)=0$ for each divisor $\delta$ and each nonprimitive divisor $d$ of $r$. A similar argument applied to $f_{r}(m, n)$ as a function of $n$ shows that $\alpha_{r}(d, \delta)=0$ unless both $d$ and $\delta$ are primitive. This completes the proof.

The following lemma, proved in [3, Lemma 3], will be used in proving the next theorem:

Lemma 8. If $r$ is primitive (free of square divisors $>1$ ), then

$$
c(n, r)=\sum_{d \mid r ;(d, n)=1} d \mu(d) \phi\left(\frac{r}{d}\right) .
$$

THEOREM 13. If $f_{r}(m, n)$ is a primitive function $(\bmod r)$ defined by (7.1), then $f_{r}(m, n)$ has the arithmetical representation

$$
\begin{aligned}
f_{r}(m, n)= & \sum_{d|\gamma(r), \delta| \gamma(r) ;(d, m)=(\delta, n)=1} d \delta \mu(d) \mu(\delta) \\
& \cdot \sum_{D|\gamma(r) / d ; E| \gamma(r) / \delta} \alpha_{r}(D d, E \delta) \phi(D) \phi(E) .
\end{aligned}
$$

Proof. From (7.1) and (7.4) one obtains

$$
\begin{aligned}
f_{r}(m, n)= & \sum_{d|\gamma(r) ; \delta| \gamma(r)} \alpha_{r}(d, \delta) \sum_{D \mid d ;(D, m)=1} D \mu(D) \phi\left(\frac{d}{D}\right) \sum_{E \mid \delta ;(E, n)=1} E \mu(E) \phi\left(\frac{\delta}{E}\right) \\
= & \sum_{D|\gamma(r) ; E| \gamma(r) ;(D, m)=(E, n)=1} D E \mu(D) \mu(E) \\
& \cdot \sum_{d|\gamma(r) ; \delta| \gamma(r) ; D|d, E| \delta} \alpha_{r}(d, \delta) \phi\left(\frac{d}{D}\right) \phi\left(\frac{\delta}{E}\right) ;
\end{aligned}
$$

hence with $d=D D^{\prime}, \delta=E E^{\prime}$, the latter formula becomes (7.5) with $d, \delta, D, E$ replaced by $D, E, D^{\prime}, E^{\prime}$ respectively. The theorem is proved.

As an application we shall obtain a particularly simple arithmetical representation of the function $\omega_{r}(m, n)$ defined in the Introduction. First we prove

Lemma 9. If $r$ is primitive and $D, E$ are divisors of $r$, then

$$
\sum_{[d, \delta]=r ; D|d, E| \delta} \mu(d) \mu(\delta)=\left\{\begin{array}{cc}
\mu(r / D) & \text { if } D=E, \\
0 & \text { otherwise. }
\end{array}\right.
$$

Proof. In view of the factorability of $\mu(r)$ it suffices to prove (7.6) for $r=p$ ( $p$ prime), $D=p^{a}, E=p^{b}$ ( $a, b$ either 0 or 1 ). In these four cases, (7.6) is easily verified, so that the lemma is true in all cases.

In addition we note some simple properties of $J(r)$. In particular, $J(r)$ is a factorable function of $r$; moreover,

$$
\sum_{d \mid \boldsymbol{r}} J(d)=r^{2}, \quad J(r)=\left(\frac{r}{\gamma(r)}\right)^{2} J(\gamma(r)) .
$$


These facts follow from (2.1) since $J(r)=J_{2}(r)$.

ThEOREM 14. If $\omega_{r}(m, n)$ is defined as in $\$ 1$, then

$$
\omega_{r}(m, n)=\phi^{2}(r) \sum_{d \mid r ;(d, m n)=1} \frac{\mu(d)}{\phi^{2}(d)} .
$$

Proof. On the basis of the definitions of $M_{r}^{s, t}(m, n)$ and $N_{r}^{s, t}(m, n)$ in $\S 6$, it is evident that $\omega_{r}(m, n)=M_{r}^{1,1}(m, n)=N_{r}^{1,1}(m, n)$. Therefore, by either Theorem 10 or $11, \omega_{r}(m, n)$ has an expansion (7.1) with

$$
\alpha_{r}(d, \delta)=\frac{\phi^{2}(r) J(r)}{r^{2}}\left(\frac{\mu(d) \mu(\delta) \mu(\Delta)}{\phi(d) \phi(\delta) J(\Delta)}\right), \quad \Delta=[d, \delta] .
$$

Thus by Theorem 13 and (7.9),

$$
\begin{aligned}
\omega_{r}(m, n)= & \frac{\phi^{2}(r) J(r)}{r^{2}} \sum_{d|\gamma(r) ; \delta| \gamma(r) ;(d, m)=(\delta, n)=1} d \delta \mu(d) \mu(\delta) \\
& \cdot \sum_{D|\gamma(r) / d ; E| \gamma(r) / \delta ; \Delta=[D d, E \delta]} \frac{\mu(D d) \mu(E \delta) \mu(\Delta) \phi(D) \phi(E)}{\phi(D d) \phi(E \delta) J(\Delta)} .
\end{aligned}
$$

With $D_{1}=d D, E_{1}=\delta E$, it follows, since $(D, d)=(E, \delta)=1$ and since $\phi(r)$ is factorable, that

$$
\begin{aligned}
\omega_{r}(m, n)= & \frac{\phi^{2}(r) J(r)}{r^{2}} \sum_{d|\gamma(r), \delta| \gamma(r) ;(d, m)=(\delta, n)=1} \frac{d \delta \mu(d) \mu(\delta)}{\phi(d) \phi(\delta)} \\
& \cdot \sum_{\Delta|\gamma(r) ; d| \Delta ; \delta \mid \Delta} \frac{\mu(\Delta)}{J(\Delta)} \sum_{\Delta=\left[D_{1}, E_{1}\right] ; d\left|D_{1}, \delta\right| E_{1}} \mu\left(D_{1}\right) \mu\left(E_{1}\right) .
\end{aligned}
$$

Application of Lemma 9 yields

$$
\omega_{r}(m, n)=\frac{\phi^{2}(r) J(r)}{r^{2}} \sum_{d \mid \gamma(r) ;(d, m n)=1} \frac{d^{2}}{\phi^{2}(d)} \sum_{\Delta|\gamma(r) ; d| \Delta} \frac{\mu(\Delta)}{J(\Delta)} \mu\left(\frac{\Delta}{d}\right) .
$$

By the factorability of $\mu(r)$ and $J(r)$, one obtains, on placing $\Delta=d \delta$ and multiplying by $J(\gamma(r)) / J(\gamma(r))$,

$$
\begin{aligned}
\omega_{r}(m, n) & =\frac{\phi^{2}(r) J(r)}{r^{2}} \sum_{d \mid \gamma(r) ;(d, m n)=1} \frac{d^{2} \mu(d)}{\phi^{2}(d) J(d)} \sum_{\delta \mid \gamma(r) / d} \frac{1}{J(\delta)} \\
& =\frac{\phi^{2}(r) J(r)}{r^{2} J(\gamma(r))} \sum_{d \mid \gamma(r) ;(d, m n)=1} \frac{d^{2} \mu(d)}{\phi^{2}(d)} \sum_{\delta \delta^{\prime}=\gamma(r) / d} J\left(\delta^{\prime}\right) .
\end{aligned}
$$

Hence, applying the first part of (7.7), it follows that

$$
\omega_{r}(m, n)=\frac{\phi^{2}(r) J(r) \gamma^{2}(r)}{r^{2} J(\gamma(r))} \sum_{d \mid r ;(d, m n)=1} \frac{\mu(d)}{\phi^{2}(d)},
$$


and (7.8) results by the second part of (7.7). The theorem is proved.

Let $r=p^{t}$, $p$ prime, $t>0$. A simple computation based on (7.8) shows that

$$
\omega_{p^{t}}(m, n)=\left\{\begin{array}{l}
p^{2(t-1)}(p-1)^{2} \\
p^{2 t-1}(p-2)
\end{array}\right.
$$

if $p \mid m n$,

if $p \nmid m n$.

Hence $\omega_{p^{t}}(m, n)=0$ if and only if $p=2$ and $m n$ is odd. By considerations of factorability we obtain immediately the following criterion for the vanishing of $\omega_{r}(m, n)$.

THeOREM 15. $\omega_{r}(m n, n)=0$ if and only if $r$ is even and $m$ and $n$ are both odd.

8. Totally primitive functions $(\bmod r)$. We have the following analogue of Theorem 12.

THEOREM 16. A function $f_{r}(m, n)$ is a totally primitive function of $m, n(\bmod$ $r)$ if and only if it possesses a representation of the form

$$
f_{r}(m, n)=\sum_{d \mid \gamma(r)} \alpha_{d}(r) c_{d}(m, n) .
$$

Proof. Certainly any function of the form (8.1) is totally primitive $(\bmod r)$. Conversely, if $f_{r}(m, n)$ is totally primitive $(\bmod r)$, it possesses representations of the form (4.5) and (7.1). But in (4.5), $\alpha_{r}(d)=0$ for each nonprimitive $d$, on the basis of Lemma 4 and the uniqueness of the coefficients in (7.1). This completes the proof.

We next prove an arithmetical formula for the expansion of totally primitive functions $(\bmod r)$. This relation is based on the following lemma which is analogous to [4, Lemma 1 and Corollary 4]. The details are omitted.

Lemma 10. The sum

$$
Q_{r}(m, n)=\sum_{(a, b, r)=1} c_{r}(m-a, n-b)
$$

has the evaluations,

$$
Q_{r}(m, n)=J(r) \sum_{d \mid r ;(d, m, n)=1} \frac{d^{2}}{J(d)} \mu\left(\frac{r}{d}\right)=\mu(r) c_{r}(m, n) .
$$

Let $f_{r}(m, n)$ be a totally even function $(\bmod r)$ defined by $(4.5)$, and place

$$
f_{r}^{*}(m, n)=\sum_{(a, b, r)=1} f_{r}(m-a, n-b) .
$$

We prove now

THEOREM 17. The function $f_{r}^{*}(m, n)$ is totally primitive $(\bmod r)$, and

$$
f_{r}^{*}(m, n) / J(r)=\sum_{d \mid r ;} \frac{d^{2}}{J(d, m, n)=1} \sum_{\delta \mid r / d} \alpha_{r}(d \delta) \mu(\delta)=\sum_{d \mid r}\left(\frac{\alpha_{r}(d) \mu(d)}{J(d)}\right) c_{d}(m, n),
$$


where $\alpha_{r}(d)$ is defined by (4.6).

Proof. By (4.5) it follows that

$$
f_{r}^{*}(m, n)=\sum_{d \mid r} \alpha_{r}(d) \sum_{(a, b, r)=1} c_{d}(m-a, n-b),
$$

so that by Remark $1(\S 2)$,

$$
f_{r}^{*}(m, n)=J(r) \sum_{d \mid r} \frac{\alpha_{r}(d)}{J(d)} Q_{d}(m, n) .
$$

Successive replacement of $Q_{d}(m, n)$ in (8.5) by its arithmetical evaluations in (8.2) leads to the theorem, with a slight computation omitted (cf. [4, Theorem 6]).

Before applying Theorem 17 we prove

Lемма 11. If $e>0$, then

$$
\sum_{d \mid r} c_{e}^{(k)}\left(\frac{r}{d}\right) \mu(d)=\left\{\begin{array}{c}
r^{k} \mu(e / r) \\
0
\end{array}\right.
$$

$$
\begin{aligned}
& \text { if } r \mid e, \\
& \text { if } r \nmid e .
\end{aligned}
$$

Proof. By (2.10), if the left member of (8.6) is denoted by $B_{r}(k, e)$, one obtains

$$
B_{r}(k, e)=\sum_{d \mid r} \mu(d) \sum_{D \mid(r / d, e)} D^{k} \mu\left(\frac{e}{D}\right)=\sum_{D \mid(r, e)} D^{k} \mu\left(\frac{e}{D}\right) \sum_{d \mid r / D} \mu(d),
$$

and the lemma follows by the characteristic property of $\mu(r)$.

We now obtain an arithmetical evaluation of the function $L_{r}^{s, t}(m, n)$ in the case $t=1(\S 6)$. Place $\theta_{r, s}(m, n)=L_{r}^{s, 1}(m, n)$, so that $\theta_{r}(m, n)=\theta_{r, 1}(m, n)$, as defined in the Introduction.

THEOREM 18. If $s \geqq 1$, then

$$
\theta_{r, s}(m, n)=\frac{J(r)}{r^{2-2 s}} \sum_{d \mid r ;(d, m, n)=1} \frac{\mu(d)}{d^{2-2} J(d)} .
$$

Proof. Clearly

$$
\begin{aligned}
\theta_{r, s}(m, n) & =\sum_{m \equiv a+b(\bmod r) ; n \equiv a^{\prime}+b^{\prime}(\bmod r)} G_{r, s}\left(a, a^{\prime}\right) \rho_{r}\left(b, b^{\prime}\right) \\
& =\sum_{((a, b), r)=1} G_{r, s}(m-a, n-b) .
\end{aligned}
$$

By Theorem 17, and (6.2a) it follows then that

$$
\theta_{r, 8}(m, n)=\frac{J(r)}{r^{2}} \sum_{d \mid r ;(d, m, n)=1} \frac{d^{2}}{J(d)} \sum_{\delta \mid r / d} c_{r}^{(2 s)}\left(\frac{r / d}{\delta}\right) \mu(\delta),
$$


but by Lemma 11, the inner sum has the value $\mu(d)(r / d)^{2 s}$. This proves the theorem.

Corollary $18.1(s=1)$.

$$
\theta_{r}(m, n)=J(r) \sum_{d \mid r ;(d, m, n)=1} \frac{\mu(d)}{J(d)}=\sum_{d \mid r ;(d, m, n)=1} C\left(\frac{r}{d}, r\right) .
$$

In case $r=p^{t}$ ( $p$ prime, $t>0$ ), it follows quite simply that

$$
\theta_{p^{t}}(m, n)= \begin{cases}p^{2(t-1)}\left(p^{2}-1\right) & \text { if } p \mid(m, n), \\ p^{2(t-1)}\left(p^{2}-2\right) & \text { if } p \nmid(m, n) .\end{cases}
$$

Hence $\theta_{p^{t}}(m, n)>0$; consequently by factorability considerations we have

Corollary 18.2. For all $m, n, r, \theta_{r}(m, n)>0$; that is, the congruence (1.1) is always solvable in $u, v, x, y$ such that $(u, v, r)=(x, y, r)=1$.

9. Infinite series expansions. For real $s$ and $n>0$, let $\sigma_{\varepsilon}(n)$ denote the sum of the $s$ th powers of the divisors of $n$, and let $\phi_{s}(n)$ denote the generalized totient,

$$
\phi_{s}(n)=\sum_{d \mid n} d^{s} \mu\left(\frac{n}{d}\right), \quad \sum_{d \mid n} \phi_{s}(d)=d^{s} .
$$

We develop trigonometric series expansions for $\sigma_{s}((m, n)) /(m, n)^{8}$ and $\phi_{s}((m, n)) /(m, n)^{s}$, analogous to Ramanujan's well-known developments of $\sigma_{s}(n) / n^{s}$ and $\phi_{s}(n) / n^{s}$. The proofs will be based on the finite Fourier expansions (Theorem 19) of the totally even functions $(\bmod r)$ defined by

$$
\sigma_{r, 8}^{*}(m, n)=\sum_{d \mid(m, n, r)} d^{2} \phi_{s}\left(\frac{r}{d}\right)
$$

and

$$
\phi_{r, s}^{*}(m, n)=\frac{\phi_{s}((m, n, r))}{(m, n, r)^{s}}=\sum_{d \backslash(m, n, r)} \frac{\mu(d)}{d^{s}} .
$$

First we note $[2,(20)]$ that

$$
\sum_{d \mid r} C_{d}(n)= \begin{cases}r^{2} & \text { if } r \mid n, \\ 0 & \text { if } r \nmid n .\end{cases}
$$

\section{THEOREM 19.}

$$
\begin{aligned}
& \sigma_{r, 8}^{*}(m, n)=r^{s} \sum_{d \mid r} \frac{c_{d}(m, n)}{d^{s}} \\
& \phi_{r, 8}^{*}(m, n)=\frac{\phi_{s+2}(r)}{r^{s+2}} \sum_{d \mid r}\left(\frac{\mu(d)}{\phi_{s+2}(d)}\right) c_{d}(m, n) .
\end{aligned}
$$


Proof. With $f_{r}(m, n)=\sigma_{r, 8}^{*}(m, n)$ in (4.5) we have by (4.6) and (9.2), in connection with (9.4),

$$
\begin{aligned}
\alpha_{r}(d) & =\frac{1}{r^{2}} \sum_{\delta \mid r}\left(\sum_{D \mid r / \delta} D^{2} \phi_{s}\left(\frac{r}{D}\right)\right) C_{\delta}\left(\frac{r}{d}\right)=\frac{1}{r^{2}} \sum_{D \mid r} D^{2} \phi_{s}\left(\frac{r}{D}\right) \sum_{\delta \mid r / D} C_{\delta}\left(\frac{r}{d}\right) \\
& =\sum_{D \mid r ; D=d e} \phi_{s}\left(\frac{r}{D}\right)=\sum_{c \mid r / d} \phi_{s}\left(\frac{r / d}{e}\right),
\end{aligned}
$$

so that by (9.1), $\alpha_{r}(d)=r^{s} / d^{s}$. This proves (9.5).

In the proof of $(9.6)$, we use $f_{r}(m, n)=\phi_{r, s}^{*}(m, n)$ in (4.5) and, by the same argument, find

$$
\alpha_{r}(d)=\frac{1}{d^{s+2}} \sum_{e \mid r / d} \frac{\mu(d e)}{e^{s+2}}=\frac{\mu(d)}{d^{s+2}} \sum_{e \mid r / d ;(d, e)=1} \frac{\mu(e)}{e^{s+2}} .
$$

Application of $[3,(7.9)]$ and the fact that $\phi_{s}\left(r_{1} r_{2}\right)=\phi_{s}\left(r_{1}\right) \phi_{s}\left(r_{2}\right)\left(r_{1}, r_{2}\right)^{\text {s }}$ $\cdot\left(\phi_{s}\left(\left(r_{1}, r_{2}\right)\right)\right)^{-1}$ (cf. [2, (13)], $\left.s=k\right)$, one obtains for $\phi_{r, s}^{*}(m, n)$,

$$
\alpha_{r}(d)=\frac{\mu(d)}{d^{s+2}}\left(\frac{\phi_{s+2}(r / d)(d, r / d)^{s+2}}{(r / d)^{s+2} \phi_{s+2}((d, r / d))}\right)=\frac{\mu(d) \phi_{s+2}(r)}{r^{s+2} \phi_{s+2}(d)} .
$$

The proof of (9.6) is complete.

The derivations of the infinite series expansions of this section are based on the following lemmas.

LemMa 12 [2, Lemma 10]. If $r_{k}$ is any sequence of positive integers such that $k \mid r_{k}(k=1,2, \cdots)$, then

$$
\lim _{k \rightarrow \infty} \sum_{d \mid r_{k}} f(d)=\sum_{m=1}^{\infty} f(m),
$$

provided the series in (9.7) converges absolutely.

Lemma 13 (cf. [2, Lemma 11]). For fixed $m, n(n>0), c_{r}(n)$ and $c_{r}(m, n)$ are bounded as functions of $r$.

We also note $[2,(43)]$ that

$$
\lim _{r \rightarrow \infty} \frac{\phi_{s}(r)}{r^{8-\epsilon}}=\infty \quad \text { for all } \epsilon>0, \quad(s>0) .
$$

Theorem 20 (cf. [2, Theorems 7 And 8]). If $n>0$ and $s>-1$, then

$$
\begin{aligned}
& \frac{\sigma_{s}((m, n))}{(m, n)^{s}}=\zeta(s+2) \sum_{r=1}^{\infty} \frac{c_{r}(m, n)}{r^{s+2}}, \\
& \frac{\phi_{s}((m, n))}{(m, n)^{s}}=\zeta^{-1}(s+2) \sum_{r=1}^{\infty}\left(\frac{\mu(r)}{\phi_{s+2}(r)}\right) c_{r}(m, n),
\end{aligned}
$$

$\zeta(t)$ denoting the Riemann $\zeta$-function. 
Proof. Replacing $s$ by $s+2$, a slight computation serves to put (9.5) in the form,

$$
\sum_{d \mid(m, n, r)} \frac{1}{d^{s}}\left(\sum_{e \mid r / d} \frac{\mu(e)}{e^{s+2}}\right)=\sum_{d \mid r} \frac{c_{d}(m, n)}{d^{s+2}} .
$$

with $r=r_{k}=k$ !, one obtains, on taking limits as $k \rightarrow \infty$,

$$
\sum_{d \mid(m, n)} \frac{1}{d^{s}}\left(\lim _{k \rightarrow \infty} \sum_{e \mid r_{k} / d} \frac{\mu(e)}{e^{s+2}}\right)=\lim _{k \rightarrow \infty} \sum_{d \mid r_{k}} \frac{c_{d}(m, n)}{d^{s+2}} .
$$

Application of Lemmas 12 and 13 as in $[2, \S 6]$ yields (9.9).

The relation (9.6) can be reformulated as

$$
\frac{\phi_{s}((m, n, r))}{(m, n, r)^{s}}=\left(\sum_{d \mid r} \frac{\mu(d)}{d^{s+2}}\right) \sum_{d \mid r}\left(\frac{\mu(d)}{\phi_{s+2}(d)}\right) c_{d}(m, n),
$$

and the procedure used in proving (9.9) can be applied in connection with (9.8) to deduce (9.10).

In case $s=0,(9.10)$ reduces to

$$
\sum_{r=1}^{\infty}\left(\frac{\mu(r)}{J(r)}\right) c_{r}(m, n)=\left\{\begin{array}{cl}
\pi^{2} / 6 & \text { if }(m, n)=1, \\
0 & \text { if }(m, n) \neq 1 .
\end{array}\right.
$$

We obtain a final expansion on the basis of the following relation, which is a consequence of (7.8) and Theorem 11, with $s=t=1$,

$$
\sum_{d \mid r ;(d, m n)=1} \frac{\mu(d)}{\phi^{2}(d)}=\frac{J(r)}{r^{2}} \sum_{e \mid r ; e=[d, \delta]}\left(\frac{\mu(e) \mu(d) \mu(\delta)}{J(e) \phi(d) \phi(\delta)}\right) c(m, d) c(n, \delta) .
$$

THEOREM 21. If $m>0, n>0$, then

$$
\sum_{d=1 ;(d, m n)=1}^{\infty} \frac{\mu(d)}{\phi^{2}(d)}=\frac{6}{\pi^{2}} \sum_{e=1 ;[d, \delta]=e}^{\infty}\left(\frac{\mu(e) \mu(d) \mu(\delta)}{J(e) \phi(d) \phi(\delta)}\right) c(m, d) c(n, \delta) .
$$

Proof. By virtue of (9.12), the proof of the theorem is similar to that of Theorem 20 , the limit on the right being justified as follows. Since $[d, \delta]$ $\geqq(d \delta)^{1 / 2}$, the series

$$
\sum_{d, \delta=1 ;[d, \delta]=e}^{\infty} \frac{1}{\left(d \delta e^{2}\right)^{1-\epsilon}}, \quad(0<\epsilon<1 / 2)
$$

converges. But for $r$ sufficiently large, $\phi(r)>r^{1-\epsilon}, J(r)=\phi_{2}(r)>r^{2-2 e}$, by (9.8), so that

$$
\sum_{d, \delta=1 ;[d, \delta]=e}^{\infty} \frac{1}{\phi(d) \phi(\delta) J(e)}
$$


converges, and hence by Lemma 13, the series on the right of (9.13) converges absoutely.

Corollary $21.1(m=n=1)$.

$$
\sum_{d=1}^{\infty} \frac{\mu(d)}{\phi^{2}(d)}=\frac{6}{\pi^{2}} \sum_{e=1 ;[d, \delta]=e}^{\infty}\left(\frac{\mu(e)}{J(e) \phi(d) \phi(\delta)}\right) .
$$

10. Generalizations. In this section we state without proof generalizations to $k$ variables of the results relating to two variables contained in Theorems 1, 2, 12, 16, and 17.

ThEOREM 22. A function $f_{r}\left(n_{1}, \cdots, n_{k}\right)$ is even $(\bmod r)$ if and only if it possesses a representation of the form,

$$
f_{r}\left(n_{1}, \cdots, n_{k}\right)=\sum_{d_{i} \mid r ; i=1, \cdots, k} \alpha_{r}\left(d_{1}, \cdots, d_{k}\right) c_{d_{1}}\left(n_{1}\right) \cdots c_{d_{k}}\left(n_{k}\right) ;
$$

moreover, the (Fourier) coefficients $\alpha_{r}\left(d_{1}, \cdots, d_{k}\right)$ are uniquely determined by

$$
\begin{aligned}
& \alpha_{r}\left(d_{1}, \cdots, d_{k}\right) \\
& \quad=\frac{1}{r^{k}} \sum_{\delta_{i} \mid r ;} \sum_{i=1, \cdots, k} f_{r}\left(\frac{r}{\delta_{1}}, \cdots, \frac{r}{\delta_{k}}\right) c_{\delta_{1}}\left(\frac{r}{d_{1}}\right) \cdots c_{\delta_{k}}\left(\frac{r}{d_{k}}\right),
\end{aligned}
$$

or by the equivalent formula,

$$
\begin{aligned}
& \alpha_{r}\left(d_{1}, \cdots, d_{k}\right) \\
& \quad=\frac{1}{r^{k} \phi\left(d_{1}\right) \cdots \phi\left(d_{k}\right)} \sum_{a_{i}(\bmod r) ; i=1, \cdots, k} f_{r}\left(a_{1}, \cdots, a_{k}\right) c_{d_{1}}\left(a_{1}\right) \cdots c_{d_{k}}\left(a_{k}\right) .
\end{aligned}
$$

THEOREM 23. An even function $f_{r}\left(n_{1}, \cdots, n_{k}\right)$ is totally even $(\bmod r)$ if and only if it has a representation of the form,

$$
f_{r}\left(n_{1}, \cdots, n_{k}\right)=\sum_{d \mid r} \alpha_{r}(d) c_{d}\left(n_{1}, \cdots, n_{k}\right) ;
$$

$\alpha_{r}(d)$ is uniquely determined by

$$
\alpha_{r}(d)=\frac{1}{r^{k}} \sum_{\delta \mid r} F_{r}\left(\frac{r}{\delta}\right) c^{(k)}\left(\frac{r}{d}\right)
$$

or by the equivalent formula,

$$
\alpha_{r}(d)=\frac{1}{r^{k} J_{k}(d)} \sum_{a_{i}(\bmod r) ; i=1, \cdots, k} f_{r}\left(a_{1}, \cdots, a_{k}\right) c_{d}\left(a_{1}, \cdots, a_{k}\right) .
$$

TheOREM 24. A function $f_{r}\left(n_{1}, \cdots, n_{k}\right)$ is primitive $(\bmod r)$ if and only if it has a representation (10.1) in which the $d_{i}$ are primitive divisors of $r$; $f_{r}\left(n_{1}, \cdots, n_{k}\right)$ is totally primitive $(\bmod r)$ if and only if it possesses a representation (10.4) in which $d$ is restricted to primitive divisors of $r$. 
Place now

$$
f_{r}^{*}\left(n_{1}, \cdots, n_{k}\right)=\sum_{\left(\left(x_{i}\right), r\right)=1} f_{r}\left(n_{1}-x_{1}, \cdots, n_{k}-x_{k}\right),
$$

where $f_{r}\left(n_{1}, \cdots, n_{k}\right)$ is defined by $(10.4)$.

THEOREM 25. The function $f_{r}^{*}\left(n_{1}, \cdots, n_{k}\right)$ is totally primitive $(\bmod r)$ and

$$
\begin{aligned}
f_{r}^{*}\left(n_{1}, \cdots, n_{k}\right) / J_{k}(r) & =\sum_{d \mid r_{i}\left(d,\left(n_{i}\right)\right)=1} \frac{d^{k}}{J_{k}(d)}-\sum_{\delta \mid r / d} \alpha_{r}(d \delta) \mu(\delta) \\
& =\sum_{d \mid r}\left(\frac{\alpha_{r}(d) \mu(d)}{J_{k}(d)}\right) c_{d}\left(n_{1}, \cdots, n_{k}\right) .
\end{aligned}
$$

\section{BIBLIOGRAPHY}

1. Eckford Cohen, A class of arithmetical functions, Proc. Nat. Acad. Sci. U.S.A. vol. 41 (1955) pp. 939-944.

2. - Trigonometric sums in elementary number theory, Amer. Math. Monthly vol. 66 (1959) pp. 105-117.

3. - Representations of even functions $(\bmod r)$, I. Arithmetical identities, Duke Math. J. vol. 25 (1958) pp. 401-421.

4. - Representations of even functions $(\bmod r)$, II. Cauchy products, Duke Math. J. vol. 26 (1959) pp. 165-182.

5. C. A. Nicol and H. S. Vandiver, A von Sterneck arithmetical function and restricted partitions with respect to a modulus, Proc. Nat. Acad. Sci. U.S.A. vol. 40 (1954) pp. 825-835.

6. K. G. Ramanathan, Some applications of Ramanujan's trigonometrical sum $c_{m}(n)$, Proc. Indian Acad. Sci. Sect. A vol. 20 (1944) pp. 62-69.

7. Albert Leon Whiteman, Finite Fourier series and equations in finite fields, Trans. Amer. Math. Soc. vol. 74 (1953) pp. 78-98.

UNIVERSITY OF TENNESSEE, KNOXVILle, TENNESSEE 